\title{
Lack of Motor Progression in Isolated Sensory Peripheral Neuropathy
}

\author{
Sameer Chhibber, Cory Toth
}

Can. J. Neurol. Sci. 2010; 37: 517-520

Nerve conduction studies (NCS) and electromyography (EMG) studies are critical for the detection, diagnosis, and characterization of peripheral neuropathy (PN). Although NCS and EMG abnormalities are often compared to clinical findings, these data are not always concordant. Examples of this discordance include patients with small fiber PN having severe deficits of nociceptive and thermal function but normal $\mathrm{NCS}^{1}$, or patients with Charcot-Marie-Tooth disease type 1A with minimal symptoms but florid NCS changes ${ }^{2,3}$. The utility of using NCS/EMG for prediction of later clinical outcomes is also unclear in many forms of $\mathrm{PN}^{4-6}$.

We prospectively identified and followed patients with isolated sensory PN (ISPN) based upon a lower limb stocking pattern of sensory loss for nociception, thermal sensation, vibration threshold and/or proprioception with initially normal motor strength, tone, reflexes and muscle bulk. The objective of this study was to determine if patients with initial ISPN go on to develop clinical and or electrophysiologic progression of motor deficits.

\begin{abstract}
MethodS
Informed consent based upon approved protocols at the University of Calgary was provided by all patients who were prospectively identified to have ISPN based upon clinical assessment by neuromuscular neurologists at the University of Calgary between January 2003 - March 2008. Patients were excluded if initial examination revealed the presence of weakness, abnormal deep tendon reflexes, abnormal tone, or muscle atrophy of intrinsic foot muscles including the extensor digitorum brevis, or if an acute onset of symptoms occurred. For inclusion, patients were required to have abnormalities in at least two sensory modalities (pinprick, temperature, vibration threshold, proprioception) in a pattern conforming to a PN. Each patient had a validated Toronto Clinical Scoring System (TCSS) score measured ${ }^{7,8}$. Prior to electrophysiological assessment (performed within one month of clinical assessment), a clinical diagnosis was assigned based upon clinical assessment and standard laboratory testing. Repeat clinical assessment, TCSS scoring, and NCS/EMG were then performed at one, two and
\end{abstract}

Table 1: Clinical characteristics of ISPN by etiology

\begin{tabular}{|c|c|c|c|c|c|c|}
\hline ETIOLOGY & Average age & Large Fiber & Small Fiber & Mixed & $\begin{array}{l}\text { Neuropathic Pain } \\
(\%)\end{array}$ & $\begin{array}{l}\text { TCSS } \\
\text { score }\end{array}$ \\
\hline $\begin{array}{l}\text { Diabetes } \\
(10 / 25)\end{array}$ & $63.3+/-11.7$ & 0 & 2 & 8 & 60 & 5.3 \\
\hline $\begin{array}{l}\text { Idiopathic } \\
(10 / 25)\end{array}$ & $64.7+/-13.1$ & 1 & 1 & 8 & 90 & 5.9 \\
\hline $\begin{array}{l}\text { Other* } \\
(5 / 25)\end{array}$ & $55.8+/-15.7$ & 0 & 1 & 4 & 20 & 4.8 \\
\hline
\end{tabular}

From the Department of Clinical Neurosciences, University of Calgary, Calgary, Alberta, Canada.

Received July 20, 2009. Final Revisions Submitted January 11, 2010.

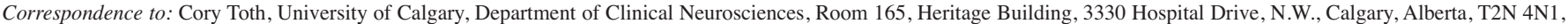
Canada. Email: corytoth@shaw.ca. 
three year follow-up assessments. Electromyography and interpretation of NCS were done by the same neuromuscular neurologist on each assessment in an unblinded fashion.

Nerve conduction studies consisted of testing using standard surface electrodes, electrode placement and limb temperature control $^{9}$. All patients had dominant upper and lower limb studied with NCS and EMG. Antidromic median, ulnar, radial, superficial peroneal and sural sensory NCS were performed for assessment of sensory nerve action potential amplitude and latency as well as sensory nerve conduction velocity. Median, ulnar, peroneal and tibial motor NCS determined compound motor action potential amplitudes and latencies, motor nerve conduction velocity, and $\mathrm{F}$ wave latencies for each nerve. All NCS data was compared to normal subject data derived within our laboratory. Sterile standard disposable concentric needle electrodes were used for EMG testing performed in tibialis anterior and gastrocnemius muscles during each yearly assessment. Electromyography scoring was assigned after assessment of a minimum of 20 separate regions within each muscle for presence of abnormal spontaneous activity (fibrillations, positive sharp waves), fasciculations, and the configuration, recruitment, and firing of voluntary motor unit potentials during minimal and maximal contraction.

Matched ANOVA testing was performed for assessment of clinical and electrophysiological data for each time point comparison, with means and standard deviations used for data presentation.

\section{Results}

\section{Clinical Progression}

A total of 25 ISPN patients (15 females) aged $62.5+/-13.0$ years were followed for three years. Three patients were only seen at zero and one year follow-up periods, and one additional patient was seen for two years follow-up - data for these four patients were not included. The average duration of PN symptoms was $6.5+/-2.1$ years. Diagnoses consisted of diabetes (10), idiopathic (10), Hepatitis C (2), monoclonal gammopathy (1), vincristine neuropathy (1), and Sjogren's syndrome (1). Concomitant neuropathic pain was present in $17 / 25(68 \%)$ patients. No statistical differences occurred in TCSS scores. The baseline, one, two and three year TCSS scores were $5.4+/-1.8$, $5.7+/-1.6,5.8+/-1.7$, and $5.8+/-1.6$ respectively, with no abnormal motor or reflex findings in any patient at follow-up. No specific treatment was given with the exception of the diabetic patients who received oral anti-hyperglycaemic agents. The specific clinical characteristics of ISPN by etiology are detailed in Table 1.

\section{Electrophysiologic Progression}

Sensory NCS (Table 2) demonstrated clear progression in all sensory nerves tested. Regardless of the underlying etiology, all patients showed a length dependant progression with all distal most nerves (superficial peroneal or sural nerve) affected at baseline and more severely affected at three year follow-up.

Motor NCS abnormalities (Table 3) were present at baseline in $24-52 \%$ of studied motor nerves. Only 5/25 (20\%) patients had entirely normal EMG and motor NCS assessments in both distal leg muscles. In all patients the initial EMG and NCS was concordant with the clinical diagnosis, abnormal spontaneous
Table 2: Average change in sensory NCS by etiology of ISPN

\begin{tabular}{llllll}
\hline ETIOLOGY & Baseline & $\mathbf{1}$ year & $\mathbf{2}$ year & 3 year & P-Value $^{* *}$ \\
\hline Idiopathic & & & & & \\
D2 median (uV) & 8.7 & 7.8 & 4.7 & 3.5 & 0.005 \\
D5 ulnar (uV) & 11.7 & 11.6 & 8.4 & 5.3 & 0.06 \\
Radial (uV) & 16.3 & 14.0 & 11.0 & 7.7 & 0.001 \\
Sup. peroneal (uV) & 1.9 & 1.7 & 0.9 & 0.7 & 0.008 \\
Sural (uV) & 3.4 & 3.0 & 1.7 & 1.5 & 0.01 \\
Diabetes & & & & & \\
D2 median (uV) & 13.7 & 11.1 & 9.3 & 8.2 & 0.0001 \\
D5 ulnar (uV) & 15.4 & 13.9 & 13.3 & 11.3 & 0.006 \\
Radial (uV) & 15.0 & 14.2 & 13.1 & 12.0 & 0.09 \\
Sup. peroneal (uV) & 3.0 & 2.6 & 2.0 & 1.5 & 0.009 \\
Sural (uV) & 3.6 & 3.1 & 2.7 & 1.8 & 0.04 \\
Other* & & & & & \\
D2 median (uV) & 13.9 & 12.3 & 11.8 & 9.1 & 0.008 \\
D5 ulnar (uV) & 10.1 & 9.3 & 9.0 & 6.5 & 0.2 \\
Radial (uV) & 26.5 & 22.8 & 23.2 & 16.6 & 0.04 \\
Sup. peroneal (uV) & 0.7 & 0.6 & 0.7 & 0.5 & 0.08 \\
Sural (uV) & 0.4 & 0.3 & 0.3 & 0.2 & 0.2 \\
\hline
\end{tabular}

*Other causes of ISPN include Hepatitis C (2), monoclonal gammopathy (1), vincristine neuropathy (1), and Sjogren's syndrome (1);

**Comparison of baseline to three-year follow-up.

activity consisting of fibrillations and PSWs occurred in 0-12\% of patients' muscles, no fasciculations were identified in any patient. Abnormalities in motor unit potentials morphology with enlarged amplitude, prolonged duration, and increased polyphasia occurred in $52-56 \%$ of muscles tested at baseline. Neuroimaging to rule out radiculopathy as a cause for these EMG changes was preformed in $32 \%(8 / 25)$ of patients, and EMG of paraspinal muscles was not routinely preformed. Despite these NCS and EMG abnormalities identified at baseline, no statistical differences occurred between baseline and follow-up studies for any of the electrophysiologic motor studies. Furthermore, patients with motor NCS or EMG abnormalities (20/25 patients) were not more likely to develop clinically evident motor abnormalities than the 20\% (5/25 patients) of our patients without such abnormalities.

Prolonged median distal motor latencies were noted in $24 \%$ of patients, and absent median sensory responses in $16 \%$ of patients versus normal ulnar motor/sensory responses. Coexistant entrapment neuropathies were examined in order to try to explain these findings, but a concomitant median neuropathy at the wrist was found in only two patients with idiopathic ISPN, and one patient with diabetic ISPN. Mild ulnar neuropathy at the elbow was seen in two patients with diabetic ISPN.

\section{Discussion}

When patients with acute or subacute onset of sensory neuropathy are examined, $56 \%$ of sensory neuronopathy 


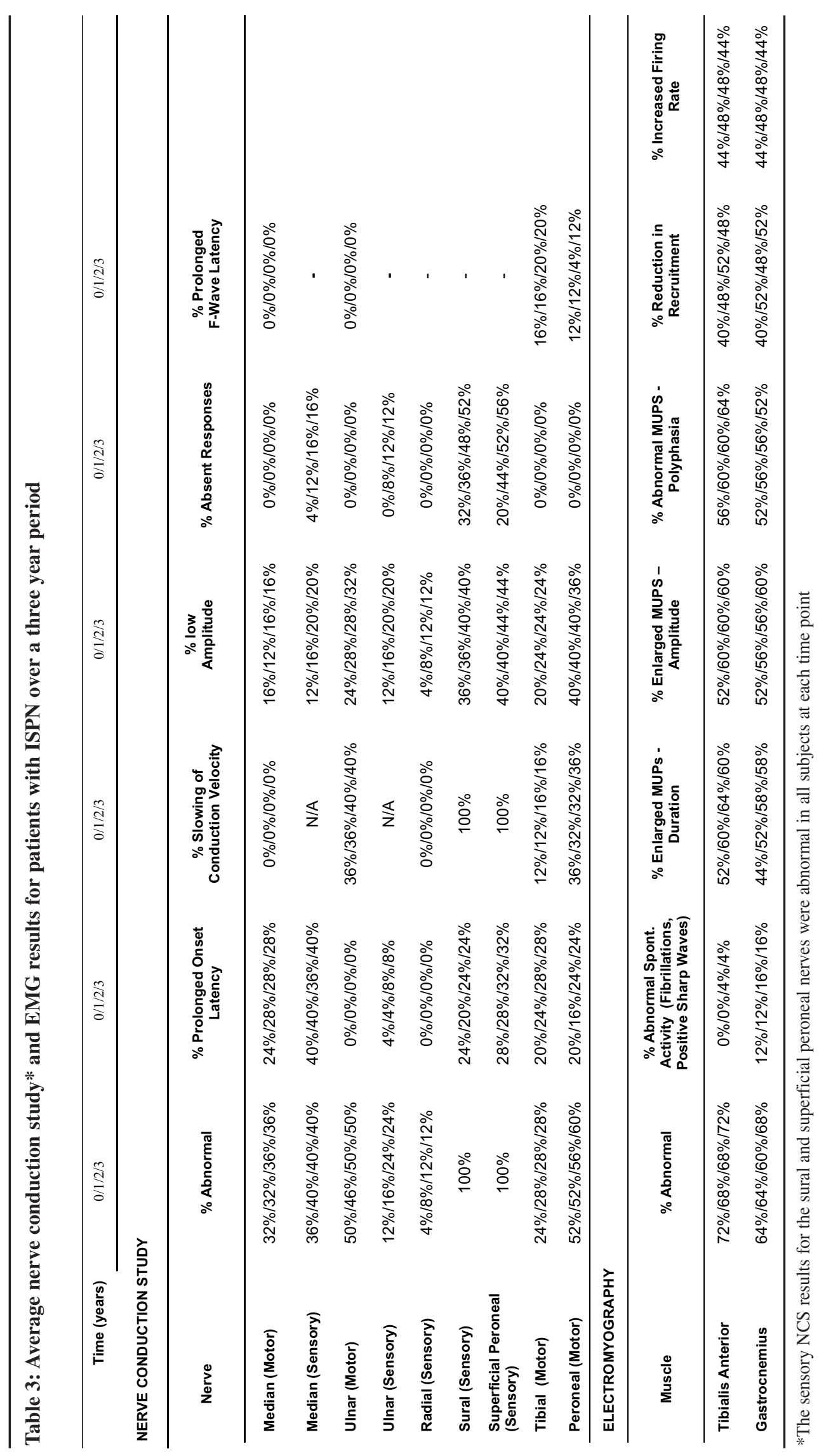


patients, and $85 \%$ of patients with ISPN have been reported to have motor NCS abnormalities, despite the absence of clinical motor deficit ${ }^{10}$. In our population of chronic ISPN patients, abnormalities in motor NCS occurred less commonly in only $60 \%$ of patients.

In our study, despite the common finding of abnormalities on motor NCS, no clinical motor or electrophysiologic motor progression was detected. In our patient population, we did not sample intrinsic foot muscles due to concerns of underlying abnormalities of spontaneous activity on EMG that have been demonstrated in $2-16 \%$ of normal control subjects ${ }^{11,12}$. For this reason, we sampled more proximal non-intrinsic leg muscles (gastrocnemius and tibialis anterior), yet still found the presence of fibrillations and PSWs in up to $12 \%$ of patients. The prevalence of motor NCS abnormalities found in our ISPN population indicates the presence of a subclinical motor neuropathy, but three years of follow-up failed to identify any accumulation of electrophysiological or clinical deficit. We postulate that in mild motor neuropathies, clinical deficits remain absent because of compensation from peripheral reinnervation and central recruitment; and the reason sensory nerve damage does lead to clinical deficits may well be that there are no similar processes for compensation.

These results suggest that, although motor electrophysiologic changes are common in ISPN, these patients do not go on to develop clinically evident motor deficits. It must be noted that our three year follow-up duration may be insufficient to detect the later development of such deficits. It is also possible that identified changes on EMG may be related to an otherwise unidentified secondary process such as radiculopathy as only $32 \%$ of our patients had neuroimaging to rule out this possibility. We also acknowledge that particular clinical etiologies will also impact upon clinical outcome of PN.

The electromyographer should be aware that patients with ISPN will frequently have abnormalities of motor NCS and EMG; yet these patients do not go on to develop clinical motor deficits at three years. And while EMG studies in ISPN do not appear to be predictive of clinical motor deficits, they remain useful in ISPN in the evaluation of common coexistant pathologies such as entrapment neuropathies and radiculopathy.

\section{REFERENCES}

1. Dyck PJ. Invited review: limitations in predicting pathologic abnormality of nerves from the EMG examination. Muscle Nerve. 1990; 115(5):371-5.

2. Dyck PJ, Lambert EH, Mulder DW. Charcot Marie Tooth disease: nerve conduction and clinical studies of a large kinship. Neurology. 1963; 13:1-11.

3. Dyck PJ, Karnes JL, Lambert EH. Longitudinal study of neuropathic deficts and nerve conduction abnormalities in hereditary motor and sensory neuropathy type 1 . Neurology. 1989; 39(10):1302-8.

4. Dyck PJ, Bushek W, Spring EM, Karnes JL, Litchy WJ, O'Brien PC, et al. Vibratory and cooling detection thresholds compared with other tests in diagnosing and staging diabetic neuropathy. Diabetes Care. 1978; 10(4):432-40.

5. Kincaid JC, Price KL, Jimenez MC, Skljarevski V. Correlation of vibratory quantitative sensory testing and nerve conduction studies in patients with diabetes. Muscle Nerve. 2007; 36(6): 821-7.

6. Stosovic M, Nikolic A, Stanojevic M, Simic-Ogrizovic S, Radovic $\mathrm{M}$, Jovanovic D, et al. Nerve conduction studies and prediction of mortality in hemodialysis patients. Ren Fail. 2008; 30(7): 6959.

7. Bril V, Perkins BA. Validation of the Toronto Clinical Scoring System for diabetic polyneuropathy. Diabetes Care. 2002; 25 (11):2048-52.

8. Perkins BA, Olaleye D, Zinman B, Bril V. Simple screening tests for peripheral neuropathy in the diabetes clinic. Diabetes Care. $2000 ; 24(2): 250-6$.

9. Daube JR. Nerve conduction studies. In: Aminoff MJ, editor. Electrodiagnosis in clinical neurology. New York: Churchhill Livingstone; 1980 . p. 229-64.

10. Camdessanché JP, Jousserand G, Ferraud K, Vial C, Petiot P, Honnorat $\mathrm{J}$, et al. The pattern and diagnostic criteria of sensory neuronopathy: a case-control study. Brain. 2009; 132(7): 1723-33.

11. Falck B, Alananta H. Fibrillation potentials, positive sharp waves and fasiculation in the intrinsic muscles of the foot in healthy subjexts. J Neurol Neurosurg Psychiatry. 1983 Jul; 46(7):681-3.

12. Gatens PF, Saeed MA. Electromyographic findings in the intrinsic muscles of normal feet. Arch Phys Med Rehabil. 1982; 63(7): 317-8. 